\title{
Impact of heart-specific disruption of the circadian clock on systemic glucose metabolism in mice
}

Tomomi Nakao, Akira Kohsaka, Tsuyoshi Otsuka, Zaw Lin Thein, Hue Thi Le, Hidefumi Waki, Sabine S Gouraud, Hayato Ihara, Masako Nakanishi, Fuyuki Sato, Yasuteru Muragaki \& Masanobu Maeda

To cite this article: Tomomi Nakao, Akira Kohsaka, Tsuyoshi Otsuka, Zaw Lin Thein, Hue Thi Le, Hidefumi Waki, Sabine S Gouraud, Hayato Ihara, Masako Nakanishi, Fuyuki Sato, Yasuteru Muragaki \& Masanobu Maeda (2017): Impact of heart-specific disruption of the circadian clock on systemic glucose metabolism in mice, Chronobiology International, DOI: 10.1080/07420528.2017.1415922

To link to this article: https://doi.org/10.1080/07420528.2017.1415922

View supplementary material ¿

曲 Published online: 22 Dec 2017.

Submit your article to this journal

Q View related articles $\asymp$

View Crossmark data $\nearrow$ 


\title{
Impact of heart-specific disruption of the circadian clock on systemic glucose metabolism in mice
}

\author{
Tomomi Nakao a, Akira Kohsakaa, Tsuyoshi Otsukaa, Zaw Lin Thein ${ }^{a}$, Hue Thi Le ${ }^{a}$, Hidefumi Wakid, \\ Sabine S Gouraude, Hayato Iharac, Masako Nakanishi' ${ }^{b}$ Fuyuki Sato ${ }^{b}$, Yasuteru Muragaki ${ }^{b}$, and Masanobu Maeda ${ }^{a}$
}

aDepartment of Physiology, Wakayama Medical University, Wakayama, Japan; bepartment of Pathology, Wakayama Medical University, Wakayama, Japan; 'Radioisotope Laboratory Center, Wakayama Medical University, Wakayama, Japan; ${ }^{\mathrm{d} G r a d u a t e}$ School of Health and Sports Science, Juntendo University, Chiba, Japan; 'Department of Biology, Faculty of Science, Ochanomizu University, Tokyo, Japan

ABSTRACT

The daily rhythm of glucose metabolism is governed by the circadian clock, which consists of cellautonomous clock machineries residing in nearly every tissue in the body. Disruption of these clock machineries either environmentally or genetically induces the dysregulation of glucose metabolism. Although the roles of clock machineries in the regulation of glucose metabolism have been uncovered in major metabolic tissues, such as the pancreas, liver, and skeletal muscle, it remains unknown whether clock function in non-major metabolic tissues also affects systemic glucose metabolism. Here, we tested the hypothesis that disruption of the clock machinery in the heart might also affect systemic glucose metabolism, because heart function is known to be associated with glucose tolerance. We examined glucose and insulin tolerance as well as heart phenotypes in mice with heart-specific deletion of Bmal1, a core clock gene. Bmal1 deletion in the heart not only decreased heart function but also led to systemic insulin resistance. Moreover, hyperglycemia was induced with age. Furthermore, heart-specific Bmal1-deficient mice exhibited decreased insulin-induced phosphorylation of Akt in the liver, thus indicating that Bmal1 deletion in the heart causes hepatic insulin resistance. Our findings revealed an unexpected effect of the function of clock machinery in a non-major metabolic tissue, the heart, on systemic glucose metabolism in mammals.
ARTICLE HISTORY

Received 6 September 2017

Revised 7 December 2017

Accepted 8 December 2017

\section{KEYWORDS}

Circadian clock; glucose metabolism; heart; liver

\section{Introduction}

Decreased heart function is associated with an increased risk of developing diabetes in humans (Demant et al. 2014; From et al. 2006; Tenenbaum et al. 2003). Both animal and clinical studies have demonstrated that chronic heart failure can cause insulin resistance (Liao et al. 2005; Nikolaidis et al. 2004; Shimizu et al. 2012; Swan et al. 1997). Heart failure results from diverse cardiovascular diseases, including coronary artery disease, hypertension, and abnormal heart valves. Some cases of heart failure have a genetic basis, such as mitochondrial DNA mutations. We and others have recently reported that a mutation in the Bmall gene, a core component of the circadian clock, also contributes to the development of chronic heart failure in mice (Durgan et al. 2011; Kohsaka et al.
2014; Lefta et al. 2012). Beyond this close relationship between the clock and heart function, clock function is also coupled to the regulation of glucose metabolism (Panda 2016; Perelis et al. 2015b; Perelis et al. 2016). These findings suggest that the circadian clock may be a key physiological system that links heart function and systemic glucose metabolism.

The mammalian circadian clock organizes daily rhythms in behaviors and biological and molecular function through highly sophisticated anatomical and molecular networks (Mohawk et al. 2012; Partch et al. 2014; Schibler et al. 2015). The suprachiasmatic nucleus (SCN) of the hypothalamus possesses the molecular clock machinery, which involves transcriptional/translational feedback loops composed of a set of core clock genes. Through neural and humoral pathways, the

CONTACT Akira Kohsaka kohsaka@wakayama-med.ac.jp E Department of Physiology, Wakayama Medical University School of Medicine, 811-1 Kimiidera, Wakayama 641-8509, Japan.

Color versions of one or more of the figures in the article can be found online at www.tandfonline.com/ICBI. 
cycling signal generated by the SCN clock is transmitted to peripheral tissues, which also possess similar molecular clock machineries to those in the SCN (Balsalobre et al. 1998; Yamazaki et al. 2000; Yoo et al. 2004). These peripheral clocks are now recognized to reside in almost all cells of the body and to be synchronized to the SCN clock, whose rhythm is entrained by environmental light/ dark cycles. Clock gene function in peripheral tissues can therefore be altered by changes in the SCN clock when the daily rhythm of rest and activity is disrupted, a condition often observed in sleep disturbances and shift work. Although these circadian disorders are associated with the dysregulation of systemic glucose metabolism (Anothaisintawee et al., 2016; Pan et al. 2011; Suwazono et al. 2009; Suwazono et al. 2010; Touma and Pannain 2011), less is known about how alterations in central and peripheral clock functions influence systemic glucose tolerance in humans. Although animal models of clock dysfunction have uncovered a tissue-specific role of clock genes in the regulation of glucose metabolism, increasing attention is being focused on only major metabolic tissues, such as the pancreas, liver, and skeletal muscle (Dyar et al. 2014; Lee et al. 2011; Marcheva et al. 2010; Perelis et al. 2015a; Petrenko et al. 2017; Rudic et al. 2004; Zhang et al. 2010). Therefore, in the present study, we sought to examine the effects of clock gene dysfunction in a non-major metabolic organ, the heart, on the regulation of systemic glucose metabolism in mice.

\section{Materials and methods}

\section{Animals}

In all experiments, we used male mice. Mice bearing a modified Bmall gene containing loxP sites

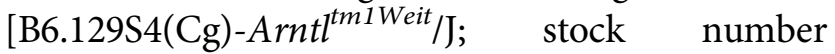
007668] and transgenic mice expressing Cre recombinase driven by the aMHC promoter [B6.FVB-Tg (Myh6-cre)2182Mds/J; stock number 011038] were obtained from Jackson Laboratory (Bar Harbor, ME, USA). Both mice were backcrossed for at least seven generations onto the C57BL/6J background and then crossed to generate heart-specific Bmal1 knockout $\left(\mathrm{H}_{-\mathrm{Bmal1}}{ }^{-/-}\right)$mice. As control mice, we used littermate animals harboring the floxed Bmall gene but no Cre transgene. All mice were housed under 12-hr light/12-hr dark (LD) cycle and maintained on regular chow diet unless otherwise noted. For diet studies, mice were placed on a low-fat (LF) $(10 \% \mathrm{kcal}$ from fat, $20 \% \mathrm{kcal}$ from protein, and $70 \% \mathrm{kcal}$ from carbohydrate; D12450B; Research Diets, Inc., New Brunswick, NJ, USA) or high-fat (HF) diet (45\% kcal from fat, $20 \% \mathrm{kcal}$ from protein, and $35 \% \mathrm{kcal}$ from carbohydrate; D12451; Research Diets, Inc.) for 8 weeks starting at 4 weeks of age. All animal care and use procedures were approved by the Wakayama Medical University Institutional Animal Care and Use Committee (Wakayama Medical University Permit Number: 743).

\section{Behavioral analysis}

For behavioral analyses, mice were housed individually in a home cage. The locomotor activity of animals was monitored for 10 days under a 12:12 LD cycle followed by 20 days in a constant darkness (DD), by using a SUPERMEX system (Muromachi Kikai Co., Ltd., Tokyo, Japan). In this system, a sensor counts the movements of the mouse by detecting the radiated body heat. Data were recorded continuously in 2-min bins with a data collection program (CompACT AMS; Muromachi Kikai Co., Ltd.). To analyze the feeding rhythm under the LD cycle, food intake was measured immediately before the onset of the dark period and immediately after the onset of the light period.

\section{Echocardiographic analysis}

Transthoracic echocardiography was performed using a $15-\mathrm{MHz}$ linear-array probe as described previously (Kohsaka et al. 2014).

\section{Blood and tissue collection}

To analyze gene expression levels in tissues, animals were sacrificed to obtain the heart, liver, epididymal fat as white adipose tissue (WAT), and soleus muscle at Zeitgeber time (ZT) 2 (ZT $0=$ lights on and ZT 12 = lights off). When the animals were dissected, blood samples were also collected by cardiac puncture. Blood samples were obtained at ZT 2 and 14. A drop of blood was used 
to measure glucose levels with a handheld glucometer (Glutest Neo; Sanwa Kagaku Kenkyusho Co., Ltd., Nagoya, Japan). The remainder of the blood sample was collected into a microtube containing EDTA and centrifuged $(7,000 \mathrm{rpm})$ for $10 \mathrm{~min}$ to obtain plasma. Both tissue and plasma samples were stored at $-80^{\circ} \mathrm{C}$ for subsequent analysis. To examine rhythmic expression of clock genes, mice were also sacrificed to obtain heart tissues at 4-hr intervals during the $24-\mathrm{hr}$ LD cycle.

\section{Primary culture}

Cardiomyocytes were isolated from 0- to 2-day-old mouse neonates with a kit from Thermo Fisher Scientific Inc. (Waltham, MA, USA). Briefly, dissected neonatal hearts were minced with fine scissors into 1 - to $3-\mathrm{mm}^{3}$ pieces and transferred to ice-cold HBSS. The minced tissues were washed twice with ice-cold HBSS to remove blood. After digestion of the minced tissues, cells were seeded on fibronectin-coated plates and cultured at a cell density of $2.5 \times 10^{5}$ cells $/ \mathrm{cm}^{2}$ in DMEM supplemented with $10 \%$ fetal bovine serum and $1 \%$ penicillin/streptomycin. After $24 \mathrm{hr}$, the medium was replaced with the same medium containing cardiomyocyte growth supplement.

\section{Glucose tolerance and insulin tolerance tests}

The intraperitoneal glucose tolerance test (IPGTT) was performed at ZT 6 following a 6-hr fast. The insulin tolerance test (ITT) was also conducted at ZT 6 but under ad libitum feeding conditions. Blood samples were collected via tail vein bleeding immediately before and at 15, 30, 60, and 120 min after i.p. injection of glucose $(2 \mathrm{~g} / \mathrm{kg})$ for IPGTT or after i.p. injection of human insulin (1 unit $/ \mathrm{kg}$ ) for ITT. Blood was immediately used for determination of glucose levels via a glucometer. Using a different set of animals, the liver tissues were also collected $60 \mathrm{~min}$ after i.p. injection of human insulin ( 1 unit $/ \mathrm{kg}$ ).

\section{Insulin infusion via the inferior vena cava and protein extraction from the liver}

After 18-hr food deprivation, mice were anesthetized with i.p. injections of ketamine $(100 \mathrm{mg} / \mathrm{kg})$ and xylazine $(10 \mathrm{mg} / \mathrm{kg})$, and insulin (5 units) or saline was injected into the inferior vena cava. The liver tissues were removed $2 \mathrm{~min}$ after the insulin injection and stored at $-80^{\circ} \mathrm{C}$ until processing. For protein extraction, stored liver tissues were homogenized in RIPA lysis buffer containing a cocktail of protease inhibitors (ATTO Co., Ltd., Tokyo, Japan). The tissue lysates were then centrifuged, and the supernatant fractions were used for immunoblot analysis.

\section{Immunoblot analysis}

Lysates were mixed with a sample preparation buffer (ATTO Co., Ltd.) and boiled for $5 \mathrm{~min}$. The boiled samples were loaded onto a 7.5\% SDS-PAGE gel (ATTO Co., Ltd.) and transferred to a membrane. Membranes were probed with primary antibodies against phosphorylated Akt (Ser473) (Cell Signaling Technology, Inc., Danvers, MA, USA) and $\beta$-actin (Cell Signaling Technology, Inc.) followed by incubation with corresponding horseradish peroxidaseconjugated secondary antibodies (Cell Signaling Technology, Inc.). The protein bands were visualized by chemiluminescence detection.

\section{RNA extraction and quantitative RT-PCR}

For tissue samples, total RNA was extracted with TRIzol RNA Isolation Reagent (Thermo Fisher Scientific Inc.). For cultured cardiomyocytes, RNeasy columns (QIAGEN, Venlo, Netherlands) were used to purify total RNA after RNA isolation by TRIzol. First-strand cDNA was synthesized using $250 \mathrm{ng}$ of cDNA and a High-Capacity cDNA Reverse Transcription kit (Thermo Fisher Scientific Inc.). Quantitative PCR was performed using a TP850 Thermal Cycler Dice Real Time system (Takara Bio, Inc., Kusatsu, Japan) as described previously (Cui et al. 2011). Gene expression levels relative to Gapdh were calculated using the comparative $C_{T}$ method. The primers used in this study are listed in Supplemental Table 1.

\section{Histology}

Tissues were fixed in $4 \%$ paraformaldehyde, processed, and embedded in paraffin. Tissue samples were then sectioned (4 microns) and stained with H\&E. 


\section{Hormone assays}

Commercially available ELISA kits were used to measure plasma insulin (Morinaga Institute of Biological Science, Inc., Yokohama, Japan) and corticosterone (AssayPro, St. Charles, MO, USA) levels. Noradrenaline levels in urine, which was collected in a vial containing $10 \mu \mathrm{l}$ of $6 \mathrm{M} \mathrm{HCl}$, were also determined by ELISA (Abnova, Taipei, Taiwan).

\section{Statistical analysis}

For comparison between two groups, an unpaired two-tailed Student's $t$-test was used to determine statistical significance. When data contained two variable factors, statistical significance was determined by two-way ANOVAs and subsequent Tukey-Kramer post hoc tests. For analysis of IPGTT and ITT data, a two-way repeated measure of ANOVA was used. In all cases, the results are presented as the means \pm SEM and were considered statistically significant at $p<0.05$.

\section{Results}

Heart-specific deficiency in the Bmal1 gene does not alter behavioral rhythms but induces a heart failure phenotype in mice

To investigate the effects of heart-specific deletion of the Bmall gene on both the heart phenotype and systemic glucose metabolism, we first validated whether the gene was exclusively knocked out in the heart of $\mathrm{H}$-Bmal1 ${ }^{-/-}$mice. Given that the heart is a heterogeneous tissue, which not only consists of cardiomyocytes but also contains interstitial cells and blood vessels, we analyzed Bmal1 expression levels in cardiomyocytes derived from neonatal $\mathrm{H}-\mathrm{Bmall}^{-/}$ - mice. We found that Bmal1 expression was significantly lower in cardiomyocytes extracted from $\mathrm{H}$-Bmal1 ${ }^{-1-}$ animals, whereas Bmal1 expression levels in major metabolic tissues (liver, WAT, and soleus muscle) were identical between control and $\mathrm{H}$-Bmal1 ${ }^{-1-}$ mice (Figure 1A). We further examined diurnal rhythms in the expression of Per2 and Reverb $\alpha$, which are core clock genes regulated by BMAL1, in the heart tissue. Rhythmic expression of these genes was significantly attenuated in $\mathrm{H}-\mathrm{Bmall}^{-/}$mice (Figure 1B), thus suggesting that the function of the molecular clock was disrupted in the hearts of

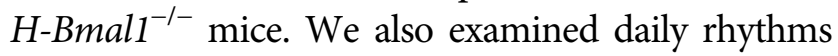
in locomotor activity and feeding behavior to evaluate the function of the central clock in $\mathrm{H}-\mathrm{Bmal1}^{-/-}$animals. We found that circadian parameters, such as the free-running period, the amplitude of the circadian rhythm, the overall activity counts, and the amount of daytime and night-time food intake, were not different between control and $\mathrm{H}$-Bmal1 ${ }^{-/-}$mice (Figure 1, $\mathrm{C}$ and D, and Supplemental Figure 1, A-C), thus indicating that the Bmall function in the brain was intact in $\mathrm{H}$-Bmall ${ }^{-1-}$ mice.

We next assessed heart function and morphology in $\mathrm{H}$-Bmal1 ${ }^{-/-}$mice. As we and others have reported previously (Durgan et al. 2011; Kohsaka et al. 2014; Lefta et al. 2012), Bmal1 ${ }^{-1-}$ hearts exhibited a hypertrophic phenotype (Figure 1E). $\mathrm{H}$-Bmal1 ${ }^{-/-}$mice also exhibited a higher ratio of heart weight to body weight than that of control animals (Figure 1F). Functional analysis by transthoracic echocardiography revealed that the left ventricular internal diameter at diastole but not systole was significantly increased in $\mathrm{H}$-Bmall $\mathrm{Bm}^{-/-}$ mice compared with control animals (Supplemental Figure 2, A and B). Furthermore, although echocardiogram analysis did not detect a significant change in fractional shortening (Supplemental Figure 2C), the expression level of $B N P$, a marker of heart failure, was significantly higher in the heart tissue of $\mathrm{H}$-Bmal1 ${ }^{-/-}$mice than in controls (Figure $1 \mathrm{G}$ ), thus indicating that heart function was decreased in $\mathrm{H}-\mathrm{Bmal1}^{-/-}$mice.

\section{H-Bmal1 ${ }^{-1-}$ mice develop hyperglycemia with age}

We next examined blood glucose levels in $\mathrm{H}$-Bmall ${ }^{-/-}$ mice. At 12 weeks of age, blood glucose levels did not differ between control and $\mathrm{H}$-Bmall ${ }^{-1-}$ mice; however, levels of blood glucose at ZT 2 but not at ZT 14 tended to be increased in $\mathrm{H}$-Bmall ${ }^{-/-}$mice compared with control animals (Figure 2A). IPGTT performed at 12 weeks of age also revealed a similar result, in which peak glucose levels after glucose injection and the area under the curve of the IPGTT were not significantly different but tended to be higher in ${\mathrm{H}-\mathrm{Bmal1}^{-/}}^{-}$mice than controls (Figure 2B). However, at 24 weeks of age, although blood glucose levels at ZT14 were not different between the 


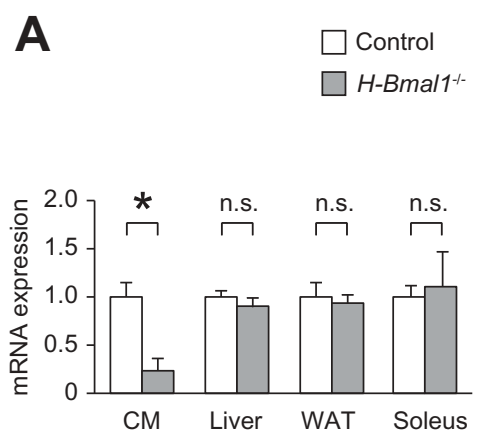

B

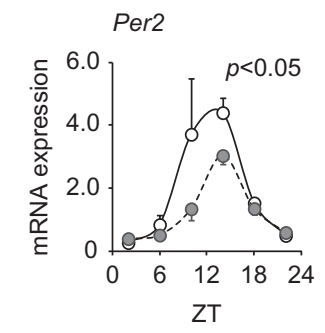

C
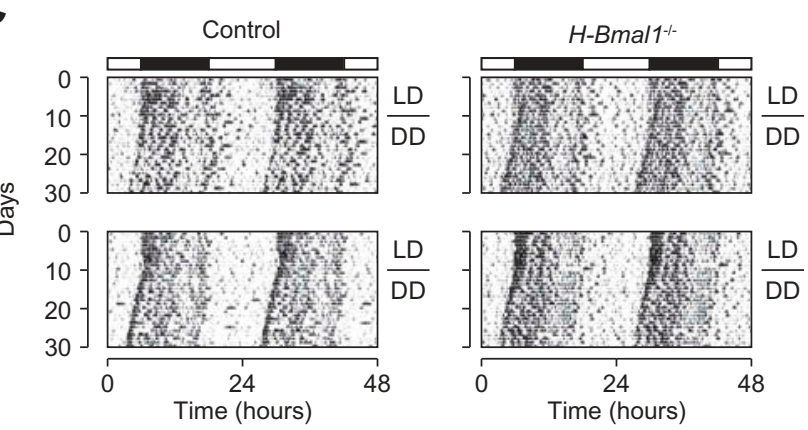

$\mathbf{E}$

$\mathbf{F}$

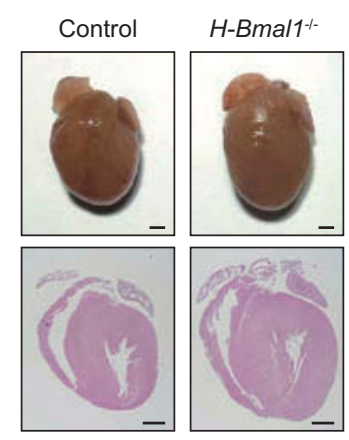

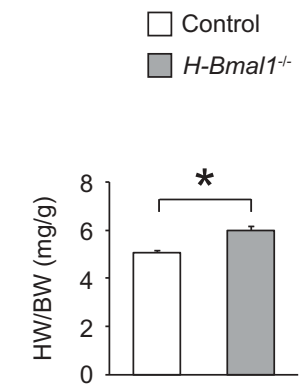

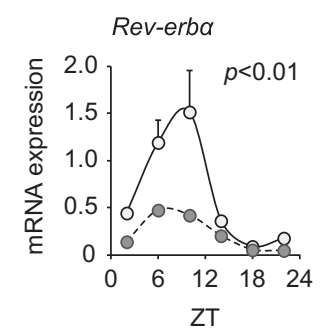

- - - Control

-- O-- H-Bmal1-1-

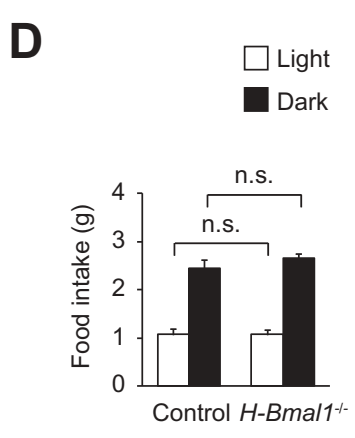

G

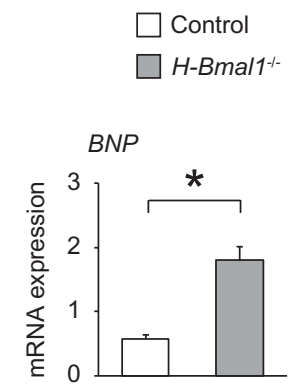

Figure 1. Heart-specific deletion of Bmal1 alters the heart phenotype without changing behavioral rhythms. (A) mRNA expression levels of Bmal1 in cardiomyocytes (CM), liver, WAT, and soleus muscle in control and $\mathrm{H}-\mathrm{Bmal1}{ }^{-/-}$mice $(n=5-10$ per group). (B) Diurnal rhythms in the mRNA expression of clock genes Per2 and Rev-erba in the heart tissue of 12-week-old animals ( $n=4$ per group per time point). (C) Two representative actograms from control (left column) and $\mathrm{H}-\mathrm{Bmal}^{-/-}$(right column) mice. Mice were maintained on a 12-hr light/dark (LD) cycle for the first 10 days and then transferred to constant darkness (DD). (D) Amount of food intake during the light and dark period ( $n=7$ per group). (E) Representative image of hearts (top panels, scale bar: $1 \mathrm{~mm}$ ) and H\&E staining of heart sections (bottom panels, scale bar: $1 \mathrm{~mm}$ ) from 12-week-old control and $\mathrm{H}$-Bmal1 ${ }^{-/-}$mice. (F) Ratios of heart weight to body weight (HW/BW) at 12 weeks of age ( $n=9-12$ per group). (G) mRNA expression levels of BNP in the heart of control and $\mathrm{H}-\mathrm{Bmal}^{-/-}$animals at 12 weeks of age ( $n=6$ per group). Data are presented as the mean \pm SEM. ${ }^{*} p<0.05$.

genotypes, those at ZT 2 were significantly higher in

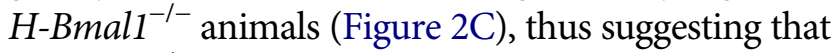
$\mathrm{H}$-Bmal1 ${ }^{-/-}$mice develop hyperglycemia with age.

\section{Loss of cardiac Bmal1 function causes insulin resistance in mice}

To explore whether the glucose intolerance observed in $\mathrm{H}_{-\mathrm{Bmall}^{-1-}}$ mice was due to insulin insufficiency and/or resistance, we examined insulin levels in the blood. We found that insulin levels were not significantly different between 12-weekold control and $\mathrm{H}$-Bmal1 ${ }^{-1-}$ mice at both ZT 2 and 14 , although insulin levels at ZT 14 tended to be higher in $\mathrm{H}$-Bmal1 ${ }^{-/-}$mice (Figure 3A). However, ITT revealed that blood glucose levels after insulin injection were not suppressed in $\mathrm{H}-\mathrm{Bmal1}^{-/-}$mice to the same level as that observed in control mice 
A

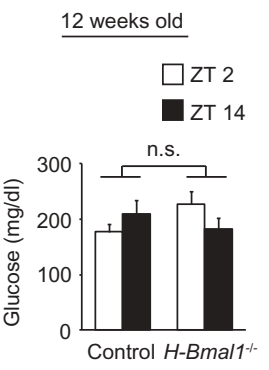

B

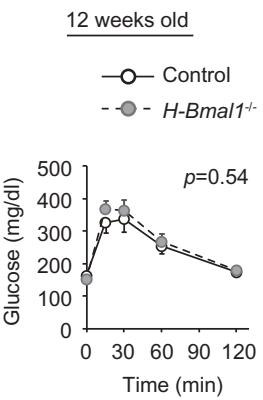

C

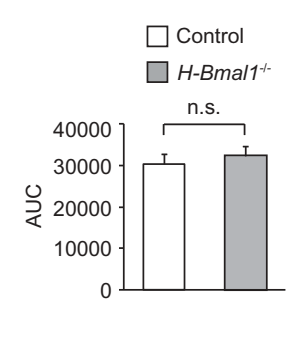

24 weeks old

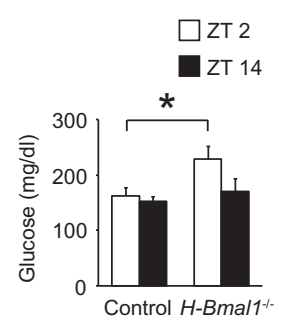

Figure 2. H-Bmal1 ${ }^{-1-}$ mice develop hyperglycemia with age. (A) Blood glucose levels at 12 weeks of age ( $n=6$ per group). (B) IPGTT: $2 \mathrm{~g} / \mathrm{kg}$ glucose was i.p. injected into $6 \mathrm{hr}$ fasted mice ( $n=10-13$ per group). Area under the curve (AUC) is quantified in the right panel. (C) Blood glucose levels at 24 weeks of age ( $n=6$ per group). Data are presented as the mean \pm SEM. ${ }^{*} p<0.05$.
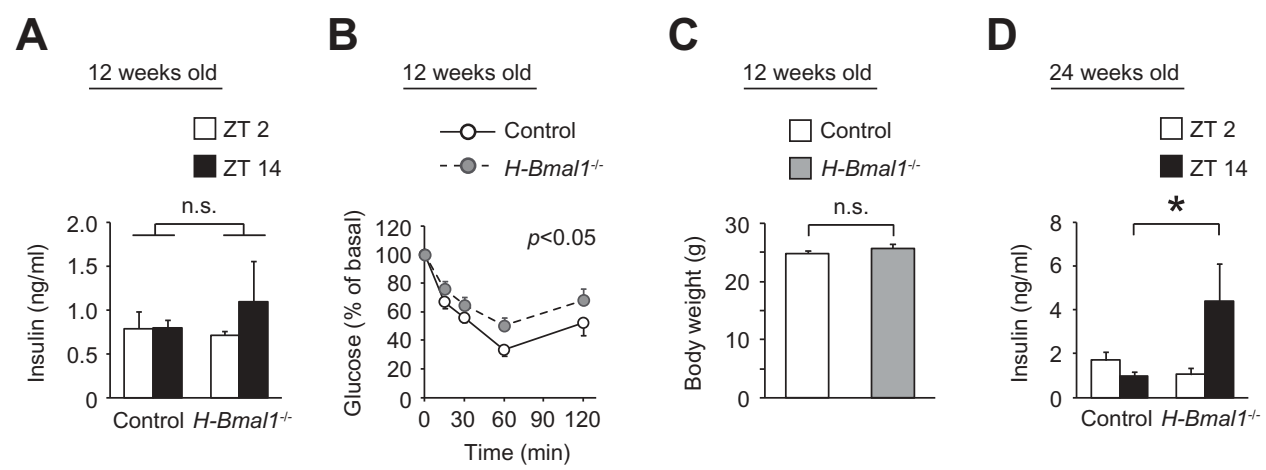

Figure 3. Loss of cardiac Bmal1 function causes insulin resistance in mice. (A) Plasma insulin levels at 12 weeks of age $(n=6$ per group). (B) ITT: 1 unit/kg insulin was i.p. injected into mice fed ad libitum ( $n=8-9$ per group). (C) Body weights at 12 weeks of age ( $n=6$ per group). (D) Plasma insulin levels at 24 weeks of age ( $n=6$ per group). Data are presented as the mean \pm SEM. ${ }^{*} p<0.05$.

(Figure 3B). This finding indicated that 12 -weekold $\mathrm{H}$-Bmall ${ }^{-/-}$mice were resistant to insulin action even before $\mathrm{H}$-Bmall ${ }^{-1-}$ mice exhibited an overt hyperglycemic phenotype (Figure 2A). Although higher body weight is often associated with insulin resistance, the body weights did not differ between control and $\mathrm{H}$-Bmall ${ }^{-1-}$ mice (Figure 3C). We also examined insulin levels at 24 weeks of age and observed a significant increase in insulin levels at ZT 14 but not at ZT 2 in $\mathrm{H}$-Bmal1 ${ }^{-/-}$animals (Figure 3D). Together, these results indicated that $\mathrm{H}$-Bmall ${ }^{-/-}$mice develop insulin resistance.

\section{HF feeding induces overt hyperglycemia in 12- week-old $\mathrm{H}$-Bmal1 ${ }^{-/-}$mice}

We further examined the effect of a HF diet on glucose metabolism in $\mathrm{H}$-Bmal1 ${ }^{-/-}$mice. Mice were fed either a LF or HF diet for 8 weeks until 12 weeks of age. HF feeding increased body weights in both control and $\mathrm{H}-\mathrm{Bmal1}^{-/-}$mice, although the increase in body weight in $\mathrm{H}$-Bmal1 ${ }^{-/-}$mice did not reach statistical significance (Figure 4A). However, interestingly, when animals were fed a HF diet, a significant increase in blood glucose levels was observed in only $\mathrm{H}$-Bmal1 ${ }^{-1-}$ mice (Figure $4 \mathrm{~B}$ ). We also examined the effects of HF feeding on plasma insulin levels and found that insulin levels were not statistically altered by a HF diet but tended to be increased in both control and $\mathrm{H}$-Bmal1 ${ }^{-1-}$ mice fed a HF diet (Figure 4C).

\section{H-Bmal1-/- mice develop hepatic insulin resistance}

Given that hepatic gluconeogenesis is a major source of glucose overproduction in insulin resistant conditions, we examined the mRNA expression levels of genes encoding key gluconeogenic enzymes, including phosphoenolpyruvate 

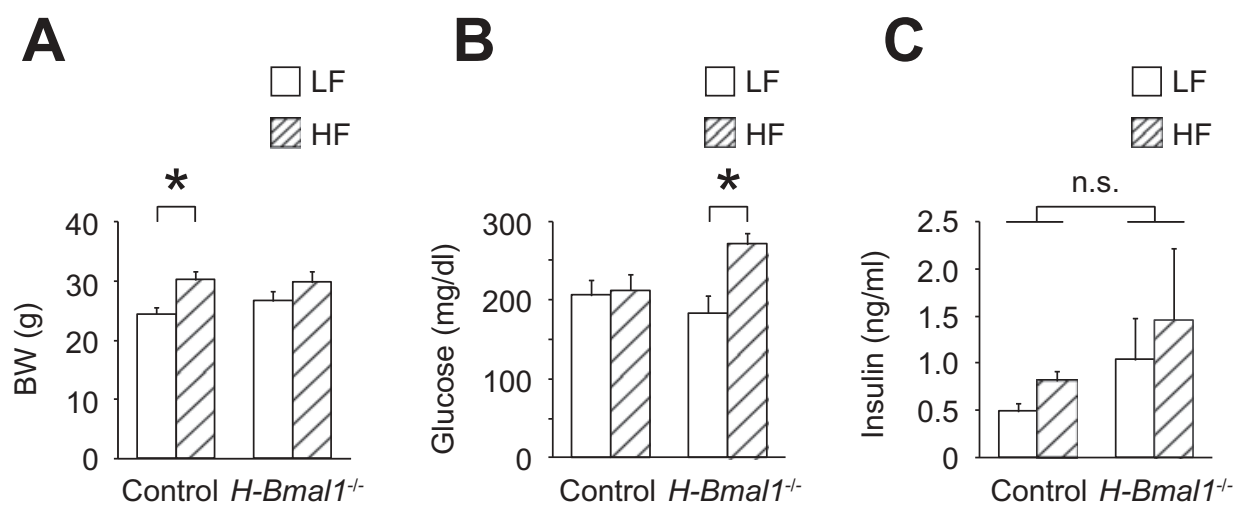

Figure 4. HF feeding induces overt hyperglycemia in $\mathrm{H}-\mathrm{Bmal1^{-1- }}$ mice at 12 weeks of age. (A) Body weights of mice placed on a lowfat (LF) or high-fat (HF) diet for 8 weeks beginning at 4 weeks of age ( $n=5-6$ per group). (B) Blood glucose and (C) plasma insulin levels for the mice in (A) after the 8-week diet study ( $n=5-6$ per group). Data are presented as the mean \pm SEM. ${ }^{*} p<0.05$.

carboxykinase (PEPCK), fructose-1,6-biphosphatase (FBPase), and glucose-6-phosphatase (G6Pase), in the liver tissues of $H$-Bmall ${ }^{-1-}$ mice. We first used the liver tissues of intact control and $H$-Bmal1 ${ }^{-1-}$ mice to analyze Pck1 (encoding cytosolic PEPCK), Fbp1 (encoding FBPase), and G6pc (encoding G6Pase) expression levels and found no significant differences in the expression of these genes between the groups (Figure 5A). However, when insulin was administered, G6Pc expression was significantly higher in $\mathrm{H}$-Bmall ${ }^{-/-}$mice than control animals; however, the expression of other gluconeogenic genes (i.e., Pck1 and Fbp1) did not differ (Figure 5B). This finding indicated that G6Pc expression was not fully suppressed by insulin in the liver of $\mathrm{H}$-Bmal1 ${ }^{-/-}$mice. $\mathrm{H}$-Bmal1 ${ }^{-/-}$ mice also showed a decreased action of insulin on the liver at the signal transduction level. We examined insulin-induced phosphorylation of Akt in the liver and observed a decrease in phosphorylated Akt levels in the livers of $\mathrm{H}$-Bmal1 ${ }^{-/-}$mice compared with control mice (Figure 5C). The reproducibility of this observation was confirmed in two independent immunoblots (Supplemental Figure 3). Collectively, these findings revealed that dysfunction of the molecular clock in the heart leads to a decreased action of insulin on hepatic glucose metabolism.

Given that heart failure is often accompanied by activation of the sympathetic nervous system, which may subsequently decrease insulin sensitivity, we analyzed levels of 24-hr urinary noradrenaline. However, we found that urinary noradrenaline levels did not differ between control and $\mathrm{H}$-Bmall ${ }^{-1-}$ mice (Figure 6A). Recent studies have demonstrated that heart failure also induces inflammation in metabolic tissues (Jahng et al. 2016; Lavine and Sierra 2017; Shimizu et al. 2012), a key feature of insulin resistance. Therefore, we examined whether these features also occurred in $\mathrm{H}$-Bmal1 ${ }^{-1-}$ mice. We first evaluated inflammatory changes by using a histological approach but did not observe any infiltration of inflammatory cells in the liver, WAT, and skeletal muscle of $\mathrm{H}$-Bmal1 ${ }^{-/}$mice (Supplemental Figure $4 \mathrm{~A}$ ). We also examined expression levels of genes associated with inflammation in these metabolic tissues. However, in agreement with the histological observation, $\mathrm{H}$-Bmal1 ${ }^{-/-}$mice did not exhibit increased expression of any inflammatory genes in the liver, WAT, and skeletal muscle (Supplemental Figure 4B). Finally, we tested the hypothesis that the insulin resistance observed in $\mathrm{H}$-Bmal1 ${ }^{-1-}$ mice was due to an increase in the blood levels of corticosterone, a major glucocorticoid in rodents. Given that plasma corticosterone levels exhibit diurnal variation, we compared corticosterone levels between the genotypes at both ZT 2 and 14. We observed no increases in plasma corticosterone levels at ZT 2 or 14 in $\mathrm{H}_{-B^{-}}$all $^{-/-}$ mice compared with control animals (Figure 6B).

\section{Discussion}

Dysfunction of the circadian clock has been proposed as one of the important causes of the development of metabolic disorders, including diabetes and dyslipidemia (Kalsbeek et al. 2014; Panda 
A

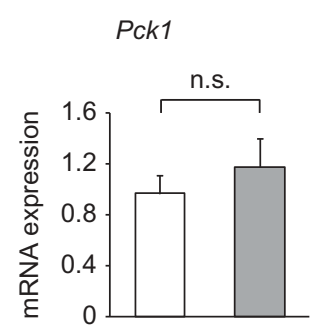

B
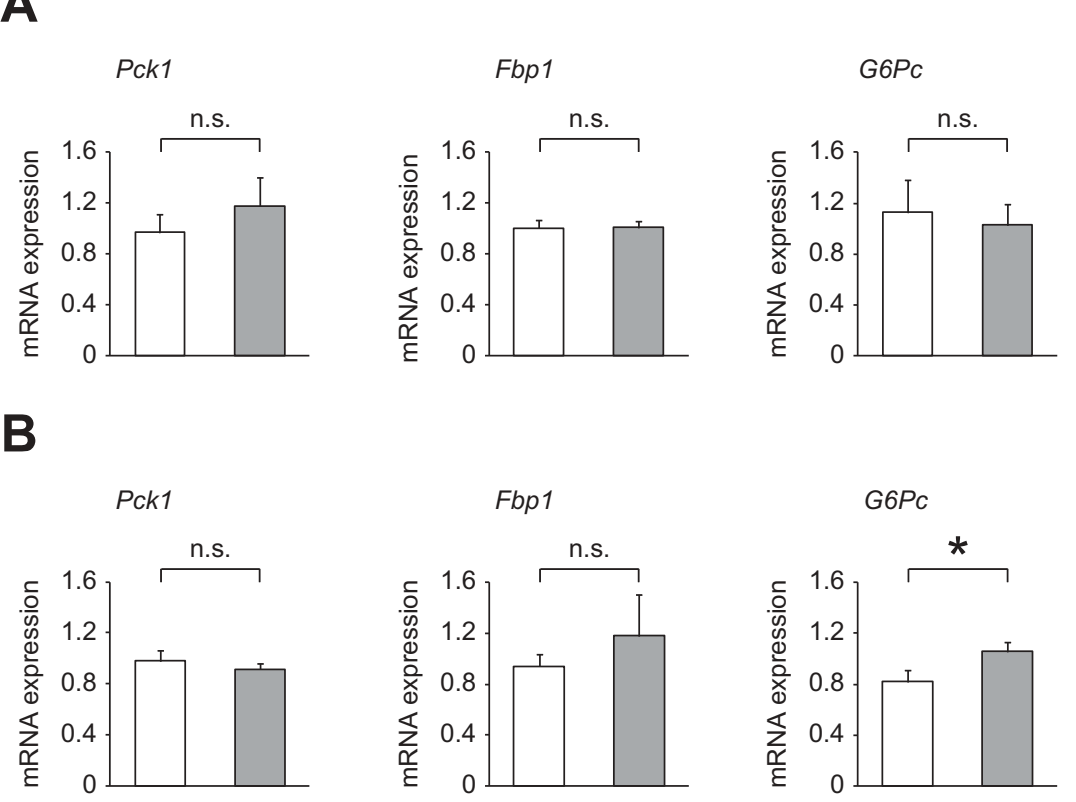

$\square$ Control

H-Bmal1 ${ }^{-1-}$

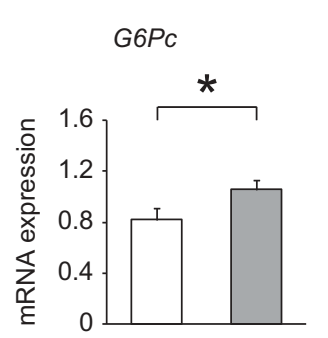

Control

H-Bmal1-1-

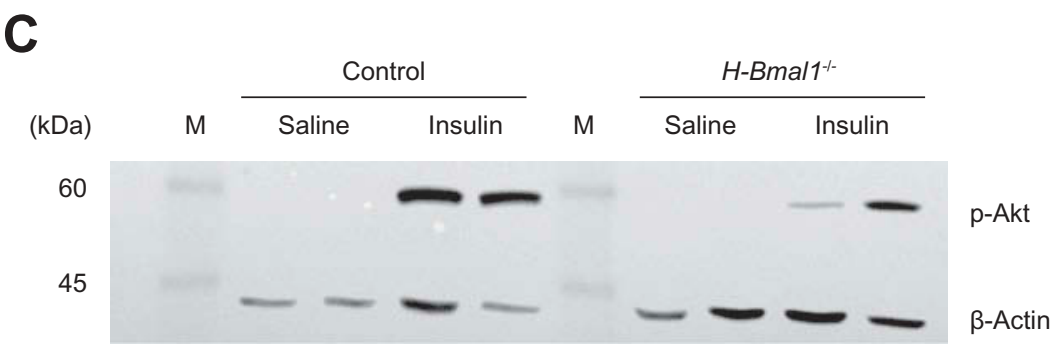

Figure 5. $\mathrm{H}-\mathrm{Bmal1}^{-/-}$mice develop hepatic insulin resistance. (A) mRNA expression levels of gluconeogenic genes in the liver of 12week-old animals ( $n=6$ per group). (B) mRNA expression levels of hepatic gluconeogenic genes 60 min after i.p. injection of insulin (1 unit $/ \mathrm{kg}$ ) ( $n=5$ per group). (C) Immunoblot analysis of insulin-stimulated phosphorylation of Akt ( $p$-Akt) in the liver. Insulin (5 units) was injected into the inferior vena cava, and tissues were collected at 2 min after the injection for protein extraction. Mice were injected with either saline or insulin. $M$ represents a marker lane. Data are presented as the mean \pm SEM. ${ }^{*} p<0.05$.

2016; Perelis et al. 2015b; Perelis et al. 2016). In humans, circadian disorders, which are frequently observed in shift work and sleep disturbances, are associated with an increased risk of impaired glucose metabolism (Anothaisintawee et al., 2016; Pan et al. 2011; Suwazono et al. 2009; Suwazono et al. 2010; Touma and Pannain 2011). This epidemiological evidence strongly suggests that the regulation of glucose metabolism is somehow linked to the function of the circadian clock. The use of animals with genetic ablation of clock gene function has further revealed that the circadian clock system indeed regulates glucose metabolism at the molecular level. For instance, mice with a dominant negative mutation in the Clock gene develop diabetes (Turek et al. 2005). Furthermore, studies using animals with tissue-specific deletion of a certain clock gene have demonstrated that pancreatic insulin secretion, hepatic gluconeogenesis, and muscular glucose transport are modulated by the molecular clocks located in the respective tissues (Dyar et al. 2014; Lee et al. 2011; Marcheva et al. 2010; Perelis et al. 2015a; Rudic et al. 2004; Zhang et al. 2010). However, studies aimed at understanding the tissue-specific roles of the molecular clock in the regulation of glucose metabolism have focused largely on metabolic tissues, such as the pancreas, liver, and skeletal muscle, and whether and how clock function in non-metabolic tissues affects systemic glucose metabolism has been less studied. Here, we demonstrated that mice with a heart-specific deletion of clock function exhibited a significantly less insulin action in the liver and consequently developing hyperglycemia with age. 


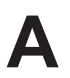

Noradrenaline

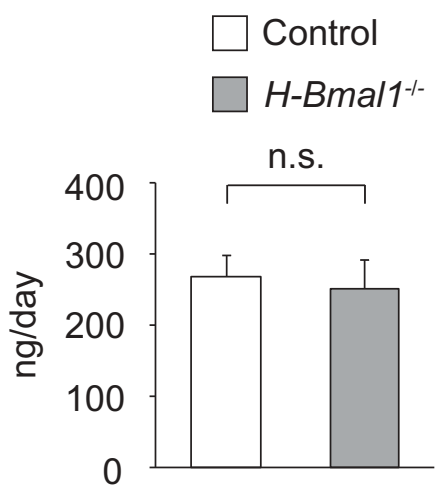

Corticosterone

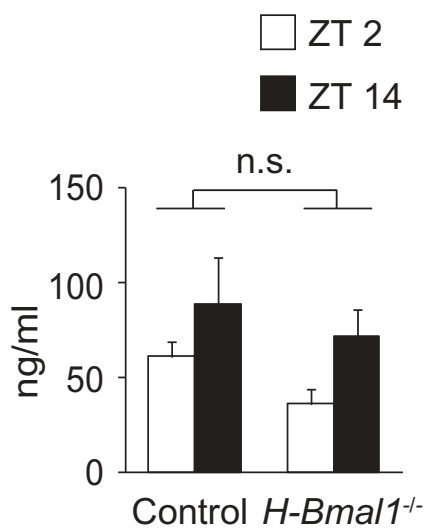

Figure 6. Insulin counterregulatory hormone levels in $\mathrm{H}-\mathrm{Bmal1}{ }^{-1-}$ mice. (A) The 24-hr urinary noradrenaline levels in 12-week-old control and $\mathrm{H}-\mathrm{Bmal}^{-1-}$ animals. The results represent averages over two consecutive days $(n=9$ per group). (B) Plasma corticosterone levels at 12 weeks of age ( $n=5-6$ per group). Data are presented as the mean \pm SEM.

The functional molecular clock is present in almost all tissues (Dibner et al. 2010; Mohawk et al. 2012), and the heart also possesses its own molecular clock, whose roles are thought to generate local cellular rhythms and regulate heart function (Durgan and Young 2010; Kohsaka et al. 2012). One of the important roles of the molecular clock in the heart appears to be the regulation of cardiac energy metabolism (Chatham and Young 2012; Duez and Staels 2010). We and others have previously reported that mice deficient in the heart clock exhibit severe defects in the molecular pathways involved in the production of cardiac bioenergy (Eckle et al. 2012; Kohsaka et al. 2014; Tsai et al. 2010). More specifically, the molecular clock is required to maintain mitochondrial structure and produce mitochondrial bioenergy in the heart (Kohsaka et al. 2014). Given that heart function relies on a continuous supply of mitochondrial bioenergy, it is understandable that mitochondrial malfunction may decrease heart function. In the present study, and in our previous report (Kohsaka et al. 2014), we observed that Bmal1 ablation in the heart leads to changes in both heart morphology and function. Moreover, although heart function can be altered by disrupted behavioral rhythms, we found that rhythms in locomotor activity and feeding behavior were identical between control and $\mathrm{H}$-Bmall ${ }^{-/}$
- mice, thus indicating that the heart failure phenotype observed in $\mathrm{H}$-Bmall ${ }^{-/-}$mice was not secondary to behavioral changes.

Although the heart is not a major organ that coordinates systemic glucose metabolism, it has long been recognized in human studies that heart failure is associated with a significant risk of developing diabetes (Demant et al. 2014; From et al. 2006; Tenenbaum et al. 2003). In addition to clinical evidence, studies using animal models of heart failure have also demonstrated that a decrease in heart function leads to insulin resistance (Liao et al. 2005; Nikolaidis et al. 2004; Shimizu et al. 2012). We found that $H$-Bmal1 ${ }^{-/-}$mice not only exhibited a heart failure phenotype but also developed impaired glucose metabolism with age. In agreement with findings from previous studies demonstrating that insulin resistance is the major cause of the impaired glucose metabolism associated with heart failure (Liao et al. 2005; Nikolaidis et al. 2004; Shimizu et al. 2012; Swan et al. 1997), we observed that $\mathrm{H}$-Bmal1 ${ }^{-/-}$mice were resistant to insulin action, and this effect became more apparent when the animals were fed a HF diet. Generally, insulin resistance involves one or more insulin target tissues, such as the liver, WAT, and skeletal muscle (Boucher et al. 2014). Recent reports have demonstrated that heart failure induced by pressure overload induces 
inflammation in WAT and thereby decreases systemic insulin sensitivity in mice (Shimizu et al. 2012). However, we did not identify any inflammatory changes in the WAT of $\mathrm{H}$-Bmall ${ }^{-/-}$mice. Instead, we found that the liver was the major organ that caused glucose intolerance in $H$-Bmal1 ${ }^{-/}$mice. The discrepancy might have been due to a difference in the use of the mouse model of heart failure (pressure overload-induced vs. genetically induced heart failure). Importantly, we found that heart-specific deletion of the Bmal1 gene decreased the insulin-induced Akt phosphorylation in the liver. Moreover, insulin did not fully suppress mRNA expression levels of $G 6 p c$, which encodes one of the key regulatory enzymes in hepatic glucose production, in the liver of $H$-Bmal1 ${ }^{-/-}$mice. These results suggested that hepatic glucose overproduction causes hyperglycemia in $\mathrm{H}$-Bmal1 ${ }^{-/-}$mice. Notably, our findings that blood glucose levels were primarily elevated during the resting (i.e., fasting) period when hepatic gluconeogenesis occurs also support the idea that the hyperglycemia observed in $\mathrm{H}$-Bmal1 ${ }^{-/-}$ mice is due to insufficient action of insulin on hepatic glucose metabolism.

As mentioned above, clinical evidence has indicated a close association between heart function and systemic glucose metabolism (Demant et al. 2014; From et al. 2006; Tenenbaum et al. 2003); however, it remains unclear how decreased heart function alters systemic glucose metabolism. Activation of the sympathetic nervous system has been proposed as the cause of insulin resistance when heart function is decreased (Riehle and Abel 2016). However, we did not identify an increase in urinary noradrenaline excretion in $\mathrm{H}$-Bmal1 ${ }^{-/-}$ mice. One plausible explanation for this observation is that the alteration in sympathetic nerve activity of $\mathrm{H}$-Bmal1 ${ }^{-/}$mice might have been too small to detect at the urinary noradrenaline level. Because the autonomic nervous system occasionally controls certain tissues (Watson et al. 2006), sympathetic efferent neurons to only a small number of tissues (i.e., liver) might be activated in $\mathrm{H}$-Bmall ${ }^{-/-}$ mice; therefore, we did not detect changes in noradrenaline at the systemic level. On the other hand, inflammation in metabolic tissues, which can cause insulin resistance, is also known to be induced under heart failure conditions (Jahng et al. 2016).
However, no inflammation was observed in the liver, WAT, and skeletal muscle of 12-week-old $\mathrm{H}$-Bmal1 ${ }^{-1-}$ mice. Finally, heart failure may also increase the blood level of corticosterone, a major insulin counterregulatory hormone in rodents, but we did not observe an increase in plasma corticosterone levels in $\mathrm{H}$-Bmal1 ${ }^{-/-}$mice.

In summary, our findings established that circadian clock function in a non-major metabolic organ, the heart, significantly influences the regulation of systemic glucose metabolism; however, the precise mechanisms remain unknown. Importantly, recent evidence that lung cancer alters the circadian regulation of liver metabolism has also emphasized the significant role of the clock in linking a pathologic condition in a given tissue and energy metabolism in other tissues (Masri et al. 2016). Providing further insight on how the function of the clock in the heart affects glucose metabolism in metabolic tissues may open new avenues through which the inter-organ communications of the circadian clock and energy metabolism may be better understood.

\section{Acknowledgements}

We thank M. Akira and other members of the Maeda laboratory for helpful discussions and technical assistance.

\section{Declaration of interest statement}

The authors declare no conflict of interest.

\section{Funding}

This study was supported by JSPS KAKENHI Grant Number 25460316 (to AK) and the Takeda Science Foundation (to $\mathrm{AK})$.

\section{References}

Anothaisintawee T, Reutrakul S, Van Cauter E, Thakkinstian A. 2016. Sleep disturbances compared to traditional risk factors for diabetes development: Systematic review and meta-analysis. Sleep Med Rev 30:11-24.

Balsalobre A, Damiola F, Schibler U. 1998. A serum shock induces circadian gene expression in mammalian tissue culture cells. Cell 93:929-37. 
Boucher J, Kleinridders A, Kahn CR. 2014. Insulin receptor signaling in normal and insulin-resistant states. Cold Spring Harb Perspect Biol 6:a009191.

Chatham JC, Young ME. 2012. Regulation of myocardial metabolism by the cardiomyocyte circadian clock. J Mol Cell Cardiol 55:139-46.

Cui H, Kohsaka A, Waki H, Bhuiyan ME, Gouraud SS, Maeda M. 2011. Metabolic cycles are linked to the cardiovascular diurnal rhythm in rats with essential hypertension. PLoS One 6:e17339.

Demant MN, Gislason GH, Kober L, Vaag A, Torp-Pedersen C, Andersson C. 2014. Association of heart failure severity with risk of diabetes: A Danish nationwide cohort study. Diabetologia 57:1595-600.

Dibner C, Schibler U, Albrecht U. 2010. The mammalian circadian timing system: Organization and coordination of central and peripheral clocks. Annu Rev Physiol 72:517-49.

Duez H, Staels B. 2010. Nuclear receptors linking circadian rhythms and cardiometabolic control. Arterioscler Thromb Vasc Biol 30:1529-34.

Durgan DJ, Tsai JY, Grenett MH, Pat BM, Ratcliffe WF, Villegas-Montoya C, Garvey ME, Nagendran J, Dyck JR, Bray MS, et al. 2011. Evidence suggesting that the cardiomyocyte circadian clock modulates responsiveness of the heart to hypertrophic stimuli in mice. Chronobiol Int 28:187-203.

Durgan DJ, Young ME. 2010. The cardiomyocyte circadian clock: Emerging roles in health and disease. Circ Res 106:647-58.

Dyar KA, Ciciliot S, Wright LE, Bienso RS, Tagliazucchi GM, Patel VR, Forcato M, Paz MI, Gudiksen A, Solagna F, et al. 2014. Muscle insulin sensitivity and glucose metabolism are controlled by the intrinsic muscle clock. Mol Metab 3:29-41.

Eckle T, Hartmann K, Bonney S, Reithel S, Mittelbronn M, Walker LA, Lowes BD, Han J, Borchers CH, Buttrick PM, et al. 2012. Adora2b-elicited Per2 stabilization promotes a HIF-dependent metabolic switch crucial for myocardial adaptation to ischemia. Nat Med 18:774-82.

From AM, Leibson CL, Bursi F, Redfield MM, Weston SA, Jacobsen SJ, Rodeheffer RJ, Roger VL. 2006. Diabetes in heart failure: Prevalence and impact on outcome in the population. Am J Med 119:591-99.

Jahng JW, Song E, Sweeney G. 2016. Crosstalk between the heart and peripheral organs in heart failure. Exp Mol Med 48:e217.

Kalsbeek A, La Fleur S, Fliers E. 2014. Circadian control of glucose metabolism. Mol Metab 3:372-83.

Kohsaka A, Das P, Hashimoto I, Nakao T, Deguchi Y, Gouraud SS, Waki H, Muragaki Y, Maeda M. 2014. The circadian clock maintains cardiac function by regulating mitochondrial metabolism in mice. PLoS One 9:e112811.

Kohsaka A, Waki H, Cui H, Gouraud SS, Maeda M. 2012. Integration of metabolic and cardiovascular diurnal rhythms by circadian clock. Endocr J 59:447-56.

Lavine KJ, Sierra OL. 2017. Skeletal muscle inflammation and atrophy in heart failure. Heart Fail Rev 22:179-89.
Lee J, Kim MS, Li R, Liu VY, Fu L, Moore DD, Ma K, Yechoor VK. 2011. Loss of Bmall leads to uncoupling and impaired glucose-stimulated insulin secretion in beta-cells. Islets 3:381-88.

Lefta M, Campbell KS, Feng HZ, Jin JP, Esser KA. 2012. Development of dilated cardiomyopathy in Bmal1-deficient mice. Am J Physiol Heart Circ Physiol 303:H475485.

Liao Y, Takashima S, Maeda N, Ouchi N, Komamura K, Shimomura I, Hori M, Matsuzawa Y, Funahashi T, Kitakaze M. 2005. Exacerbation of heart failure in adiponectin-deficient mice due to impaired regulation of AMPK and glucose metabolism. Cardiovasc Res 67:705-13.

Marcheva B, Ramsey KM, Buhr ED, Kobayashi Y, Su H, Ko $\mathrm{CH}$, Ivanova $\mathrm{G}$, Omura $\mathrm{C}$, Mo S, Vitaterna $\mathrm{MH}$, et al. 2010. Disruption of the clock components CLOCK and BMAL1 leads to hypoinsulinaemia and diabetes. Nature 466:627-31.

Masri S, Papagiannakopoulos T, Kinouchi K, Liu Y, Cervantes M, Baldi P, Jacks T, Sassone-Corsi P. 2016. Lung adenocarcinoma distally rewires hepatic circadian homeostasis. Cell 165:896-909.

Mohawk JA, Green CB, Takahashi JS. 2012. Central and peripheral circadian clocks in mammals. Annu Rev Neurosci 35:445-62.

Nikolaidis LA, Sturzu A, Stolarski C, Elahi D, Shen YT, Shannon RP. 2004. The development of myocardial insulin resistance in conscious dogs with advanced dilated cardiomyopathy. Cardiovasc Res 61:297-306.

Pan A, Schernhammer ES, Sun Q, Hu FB. 2011. Rotating night shift work and risk of type 2 diabetes: Two prospective cohort studies in women. PLoS Med 8:e1001141.

Panda S. 2016. Circadian physiology of metabolism. Science 354:1008-15.

Partch CL, Green CB, Takahashi JS. 2014. Molecular architecture of the mammalian circadian clock. Trends Cell Biol 24:90-99.

Perelis M, Marcheva B, Ramsey KM, Schipma MJ, Hutchison AL, Taguchi A, Peek CB, Hong H, Huang W, Omura C, et al. 2015a. Pancreatic beta cell enhancers regulate rhythmic transcription of genes controlling insulin secretion. Science 350:aac4250.

Perelis M, Ramsey KM, Bass J. 2015b. The molecular clock as a metabolic rheostat. Diabetes Obes Metab 17(Suppl 1):99105.

Perelis M, Ramsey KM, Marcheva B, Bass J. 2016. Circadian transcription from beta cell function to diabetes pathophysiology. J Biol Rhythms 31:323-36.

Petrenko V, Saini C, Giovannoni L, Gobet C, Sage D, Unser M, Heddad Masson M, Gu G, Bosco D, Gachon F, et al. 2017. Pancreatic $\alpha$ - and $\beta$-cellular clocks have distinct molecular properties and impact on islet hormone secretion and gene expression. Genes Dev 31:383-98.

Riehle C, Abel ED. 2016. Insulin signaling and heart failure. Circ Res 118:1151-69.

Rudic RD, McNamara P, Curtis AM, Boston RC, Panda S, Hogenesch JB, Fitzgerald GA. 2004. BMAL1 and 
CLOCK, two essential components of the circadian clock, are involved in glucose homeostasis. PLoS Biol 2:e377.

Schibler U, Gotic I, Saini C, Gos P, Curie T, Emmenegger Y, Sinturel F, Gosselin P, Gerber A, Fleury-Olela F, et al. 2015. Clock-talk: Interactions between central and peripheral circadian oscillators in mammals. Cold Spring Harb Symp Quant Biol 80:223-32.

Shimizu I, Yoshida Y, Katsuno T, Tateno K, Okada S, Moriya J, Yokoyama M, Nojima A, Ito T, Zechner R, et al. 2012. p53-induced adipose tissue inflammation is critically involved in the development of insulin resistance in heart failure. Cell Metab 15:51-64.

Suwazono Y, Dochi M, Oishi M, Tanaka K, Kobayashi E, Sakata K. 2009. Shiftwork and impaired glucose metabolism: A 14-year cohort study on 7104 male workers. Chronobiol Int 26:926-41.

Suwazono Y, Uetani M, Oishi M, Tanaka K, Morimoto H, Sakata K. 2010. Calculation of the benchmark duration of shift work associated with the development of impaired glucose metabolism: A 14-year cohort study on 7104 male workers. Occup Environ Med 67:532-37.

Swan JW, Anker SD, Walton C, Godsland IF, Clark AL, Leyva F, Stevenson JC, Coats AJ. 1997. Insulin resistance in chronic heart failure: Relation to severity and etiology of heart failure. J Am Coll Cardiol 30:527-32.

Tenenbaum A, Motro M, Fisman EZ, Leor J, Freimark D, Boyko V, Mandelzweig L, Adler Y, Sherer Y, Behar S. 2003. Functional class in patients with heart failure is associated with the development of diabetes. Am J Med 114:271-75.

Touma C, Pannain S. 2011. Does lack of sleep cause diabetes? Cleve Clin J Med 78:549-58.

Tsai JY, Kienesberger PC, Pulinilkunnil T, Sailors MH, Durgan DJ, Villegas-Montoya C, Jahoor A, Gonzalez R, Garvey ME, Boland B, et al. 2010. Direct regulation of myocardial triglyceride metabolism by the cardiomyocyte circadian clock. J Biol Chem 285:2918-29.

Turek FW, Joshu C, Kohsaka A, Lin E, Ivanova G, McDearmon E, Laposky A, Losee-Olson S, Easton A, Jensen DR, et al. 2005. Obesity and metabolic syndrome in circadian Clock mutant mice. Science 308:1043-45.

Watson AM, Hood SG, May CN. 2006. Mechanisms of sympathetic activation in heart failure. Clin Exp Pharmacol Physiol 33:1269-74.

Yamazaki S, Numano R, Abe M, Hida A, Takahashi R, Ueda M, Block GD, Sakaki Y, Menaker M, Tei H. 2000. Resetting central and peripheral circadian oscillators in transgenic rats. Science 288:682-85.

Yoo SH, Yamazaki S, Lowrey PL, Shimomura K, Ko CH, Buhr ED, Siepka SM, Hong HK, Oh WJ, Yoo OJ, et al. 2004. PERIOD2::LUCIFERASEreal-time reporting of circadian dynamics reveals persistent circadian oscillations in mouse peripheral tissues. Proc Natl Acad Sci USA 101:5339-46.

Zhang EE, Liu Y, Dentin R, Pongsawakul PY, Liu AC, Hirota T, Nusinow DA, Sun X, Landais S, Kodama Y, et al. 2010. Cryptochrome mediates circadian regulation of cAMP signaling and hepatic gluconeogenesis. Nat Med 16:1152-56. 